\title{
Uso de agonista de GnRH para inducir ovulación en ciclos de hiperestimulación ovárica controlada. Experiencia de Clínica Monteblanco, Santiago, Chile
}

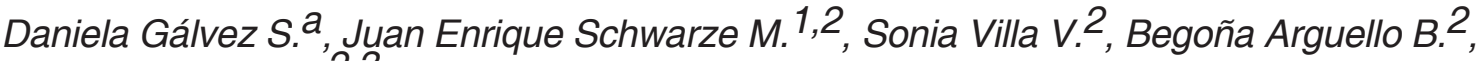 \\ Ricardo Pommer T.2,3 \\ ${ }^{1}$ Departamento Clínico de Obstetricia y Ginecología, Universidad de Santiago de Chile. ${ }^{2}$ Unidad Medicina Reproductiva \\ Clínica Monteblanco. ${ }^{3}$ Instituto de Investigación Materno Infantil de la Universidad de Chile.
}

\footnotetext{
alumna, Escuela de Medicina, Universidad de Santiago de Chile.
}

\section{RESUMEN}

Objetivo: Presentar la experiencia de la Unidad de Medicina Reproductiva de Clínica Monteblanco con el uso de análogos GnRh para la inducción final de la maduración ovocitaria. Método: Se registraron los casos de IVF/ICSI durante el año 2012 en los que se indujo la maduración final ovocitaria con análogos GnRh (Lupron $\AA$ ). Todos los ciclos fueron estimulados con FSHr (Puregon®) y gonadotrofina urinaria altamente purificada (Menopur®), para la prevención del alza prematura de LH, el día 5ํㅜ de estimulación se agregó diariamente antagonista de GnRh. La maduración ovocitaria final se realizó con 1,25 mg de acetato de leuprolide (Lupron $®)$, posteriormente se realizó aspiración folicular bajo guía ecográfica. Todos los embriones obtenidos fueron vitrificados y transferidos en ciclos posteriores. Resultados: Entre enero y diciembre del año 2012 se registraron 110 pacientes cuya inducción de maduración final ovocitaria se realizó con acetato de leuprolide. El promedio de ovocitos recuperados fue de 21, la proporción de ovocitos maduros fue de $72 \%$ y la frecuencia de fecundación fue de $64 \%$. No hubo ningún caso de síndrome de hiperestimulación ovárico severo. Conclusiones: En los casos presentados de inducción de la maduración ovocitaria final con acetato de leuprolida, los resultados obtenidos son óptimos en términos de número de ovocitos en metafase II recuperados y en frecuencia de fecundación, mostrando ser una alternativa eficiente en la prevención del síndrome de hiperestimulación ovárico severo, sin alterar el pronóstico de las pacientes.

\section{PALABRAS CLAVE: Síndrome de hiperstimulación ovárica, inducción de la ovulación, agonista GnRh}

\section{SUMMARY}

Objective: To present the experience of the Reproductive Medicine Unit of Clinica Monteblanco inducing oocyte final maturation by GnRh analogue administration. Methods: We analysed all IVF/ICSI cases performed in 2012, in which final oocyte maturation was induced by administration of GnRH analogue (Lupron®). Controlled ovarian hyperstimulation was achieved bydaily $\mathrm{rFSH}$ (Puregon ${ }^{\circledR}$ ) and highly purified urinary gonadotropin (Menopur ${ }^{\circledR}$ ) administration. In order to prevent premature LH rise, on the 5th day of stimulation daily $\mathrm{GnRH}$ antagonist (Orgalutran $囚$ ) was added. Final oocyte maturation was induced by the administration of $1.25 \mathrm{mg}$ leuprolide acetate (Lupron $\AA$ ). Follicular aspiration was subsequently performed under ultrasound guidance. All embryos were vitrified and transferred in a subsequent cycle. Results: We registered 110 patients. The mean number of recovered oocytes was 21 ; the proportion of mature oocytes was $72 \%$, 
and the fecundation rate reached was $64 \%$. No case of severe ovarian hyperstimulation syndrome (OHSS) was recorded. Conclusions: In this cohort, the use of leuprolide acetate for induce final oocyte maturation demonstrated to be an efficient alternative to induce oocyte final maturation, while preventing OHSS.

\section{KEY WORDS: Ovarian hyperstimulation syndrome, ovulation induction, GnRH analogue}

\section{INTRODUCCIÓN}

Probablemente, la complicación más temida al realizar un ciclo de fecundación in vitro (FIV) es el síndrome de hiperestimulación ovárica (SHEO), debido a la hiperestimulación ovárica controlada. El SHEO corresponde a una respuesta ovárica exagerada de origen iatrogénico que suele presentarse pocos días después de la inducción final de la ovulación $(1,2)$. Los cuadros leves y moderados de SHEO se caracterizan por escaso compromiso del estado general, habitualmente sólo dolor abdominal difuso y molestias generales, siendo autolimitados. En cambio, los cuadros severos se caracterizan por la presencia de alteraciones hemodinámicas causadas por la presencia de un tercer espacio, lo que requiere hospitalización y manejo en cuidados intermedios, con un importante riesgo vital $(1,3)$.

La frecuencia reportada de SHEO depende de la clasificación utilizada, sin embargo se estima en $1 \%$ de los ciclos de FIV $(2,3,4)$. Se han descrito múltiples estrategias para prevenir este cuadro iatrogénico. Entre las más utilizadas se encuentra la cancelación del ciclo, la suspensión temporal de la administración de gonadotrofinas exógenas, la administración intravenosa de albúmina al momento de la culdocentesis, el uso de corticoides, y la inducción final de la maduración ovocitaria con la administración de un análogo de $\operatorname{GnRH}(2,3)$.

La administración de un análogo de GnRH induce una rápida liberación de $\mathrm{LH}$ y $\mathrm{FSH}$, alcanzando niveles plasmáticos similares a los fisiológicos en el momento de la ovulación (5-8). Sin embargo, son muchos los centros que no lo usan por temor a que sea menos eficiente en el proceso de inducir la maduración final de los ovocitos.

El objetivo de este estudio es presentar la experiencia de la Unidad de Medicina Reproductiva de Clínica Monteblanco con el uso de un análogo de $\mathrm{GnRH}$, el acetato de leuprolide, para inducir la maduración final ovocitaria.

\section{MATERIAL Y MÉTODOS}

Se registraron los ciclos en los que se indujo maduración final ovocitaria mediante la administración de Lupron $®$ (acetato de leuprolide) durante el año 2012 en la Unidad de Medicina Reproductiva de Clínica Monteblanco. Es práctica habitual de nuestro grupo inducir la maduración final de ovo- citos con agonista de GnRH en donantes de ovocitos, y en las pacientes que cuenten con más de 19 folículos con diámetro promedio mayor a trece milímetros.

Hiperstimulación ovárica controlada. Se indujo hiperestimulación ovárica controlada mediante la administración diaria de FSH recombinante (Puregon®) y gonadotrofina urinaria altamente purificada (Menopur®). Al quinto día de estimulación se agregó diariamente un antagonista de GnRH (Orgalutran®) para evitar la liberación prematura de LH. El desarrollo folicular fue monitorizado mediante ecografias transvaginales seriadas, y cuando al menos dos folículos alcanzaron un diámetro promedio de $17 \mathrm{~mm}$, se indicó la administración subcutánea de $1,25 \mathrm{mg}$ de acetato de leuprolide (Leuprolide $®$ ). A las 35-37 horas de administrado el agonista de $\mathrm{GnRH}$, se recuperaron los ovocitos de los folículos mediante aspiración bajo guía ecográfica.

Procedimiento de laboratorio de embriología. Los ovocitos fueron identificados mediante una lupa, y catalogados mediante la identificación del primer polocito, como maduros (en metafase II) o inmaduros (metafase I). Luego, después de dejarlos estabilizar seis horas en medio de cultivo, fueron inyectados con un espermatozoide mediante un micromanipulador. Veinte y cinco horas luego de la inyección se comprobó la presencia de dos pronúcleos, señal inequívoca de fecundación exitosa. En ningún caso se transfirieron embriones, los que fueron vitrificados para transferir en un subsecuente ciclo cuando correspondiera.

Análisis estadístico. Se presenta la información como promedios y desviación estándar, con intervalo de confianza del $95 \%$. Para análisis multivariable se utilizó el análisis de regresión de Poisson para calcular la razón de las tasas de incidencia de ovocitos fecundados, ocupando como variable independiente la edad de la paciente y como exposición el número de ovocitos en metafase II.

\section{RESULTADOS}

Entre enero y diciembre de 2012 se registraron 110 pacientes en las que se indujo la maduración final de los ovocitos en un ciclo de hiperestimulación ovárica controlada mediante la administración de $1,25 \mathrm{mg}$ de acetato de leuprolide.

La media de la edad fue 29 años con (rango: 19 a 40 años); el $56 \%$ de las pacientes tenían menos 
de 30 años. El promedio de ovocitos recuperados fue de 21 , el promedio de ovocitos en metafase II fue de 15,1 y el promedio de ovocitos fecundados fue de 9,6 folículos por ciclo (Tabla I). De este modo, la proporción de ovocitos maduros fue de $72 \%$ (IC95\% 70-74), y la de fecundación fue de 64\% (IC95\% 62-66). Al realizar una regresión de Poisson para determinar la razón de incidencia de fecundación exitosa, encontramos que cada año extra de edad de la mujer disminuía la razón de incidencia en $8 \%$ aunque sin alcanzar significancia estadística $(p=0,569)$. Todas las pacientes menstruaron dentro de los diez días posteriores a la culdocentesis; la mayoría lo hizo a los siete días de recuperados los ovocitos. No se registró ningún casos de síndrome de hiperestimulación ovárica severa.

\section{Tabla I \\ RESULTADOS DE CICLOS DE ASPIRACIÓN EN PACIENTES A LAS QUE SE INDUJO OVULACIÓN CON AGONISTA GnRH}

\begin{tabular}{lc}
\hline Variable & Resultados \\
\hline $\begin{array}{l}\text { Número de ovocitos recuperados; } \\
\text { media (p25-p75) }\end{array}$ & 18 (14-40) \\
$\begin{array}{l}\text { Número de ovocitos metafase 2; } \\
\text { media (p25-p75) }\end{array}$ & 14 (10-32) \\
Fecundación \% (IC95\%) & 64 (62-66) \\
\hline
\end{tabular}

\section{DISCUSIÓN}

En el presente estudio, presentamos nuestra experiencia usando un agonista de $\mathrm{GnRH}$ para inducir maduración final ovocitaria. Si bien el uso de agonistas de $\mathrm{GnRH}$ se ha descrito como una alternativa eficiente para prevenir la ocurrencia de síndrome de hiperestimulación ovárica $(2,4)$, hasta donde llega nuestro conocimiento somos el único grupo que lo utiliza en forma rutinaria, por el temor de una mala respuesta de maduración ovocitaria al uso de este fármaco.

Encontramos que los resultados en términos de maduración ovocitaria y fecundación son óptimos, por lo que no se altera el pronóstico de la paciente al inducir la maduración final ovocitaria con un análogo de GnRH. Nuestros hallazgos son similares a los publicados por Shapiro y cols (9), quienes describieron una proporción de ovocitos en metafase II y de fecundación de $69 \%$ y $72 \%$, respectivamente.

Ninguna paciente desarrolló un cuadro de síndrome de hiperestimulación ovárica controlada. Aunque es importante señalar que nuestro estudio no estaba diseñado para pesquisar diferencias en la frecuencia de síndrome de hiperestimulación ovárica. Considerando que la frecuencia de este cuadro llega a $1 \%$, deberíamos esperar cerca de diez años para poder lograr alcanzar un número adecuado de eventos para poder demostrar la efectividad de la intervención. Sin embargo, el hecho que la mayoría de las pacientes presentan menstruación a los pocos días de administrado el agonista $\mathrm{GnRH}$ es sugerente de un efecto luteolítico de esta intervención, con lo que disminuirían, en forma paralela, los niveles del factor de crecimiento endotelial, principal responsable del cuadro clínico del síndrome hiperestimulación ovárica (1).

Es importante señalar que en todos los casos se difirió la transferencia embrionaria, ya que está demostrado que en ciclos en los que se ha inducido la maduración final ovocitaria mediante la administración de algún agonista de $\mathrm{GnRH}$, la tasa de implantación embrionaria cae en forma significativa $(4,5,9)$. Dado que la menstruación ocurrió en menos de dos semanas, la transferencia embrionaria sólo se ve postergada en alrededor de 10 días, lo que ayuda a manejar la ansiedad de las pacientes que no quieren posponer su tratamiento.

\section{CONCLUSIÓN}

Esperamos que nuestra experiencia aliente a otros centros a comenzar el uso de análogo de GnRH para inducir maduración final de ovocitos en pacientes sometidas a hiperestimulación ovárica controlada, en riesgo de desarrollar síndrome de hiperestimulación ovárica, aumentando así la seguridad de las pacientes sometidas a estos tratamientos.

\section{REFERENCIAS}

1. Shmorgun D, Claman P. Joint Society of Obstetricians and Gynaecologists of Canada-Canadian Fertility Andrology Society Clinical Practice Guidelines Committee; Reproductive Endocrinology and Infertility Committee of the SOGC; Executive and Council of the Society of Obstetricians; Gynaecologists of Canada; Board of the Canadian Fertility and Andrology Society, The diagnosis and management of ovarian hyperstimulation syndrome. J Obstet Gynaecol Can 2011;33:1156-62.

2. Delvigne A, Rozenberg S. Epidemiology and prevention of ovarian hyperstimulation syndrome (OHSS): a review. Human Reprod Update 2002;8:559-77.

3. Alper MM, Smith LP, Sills ES. Ovarian hyperstimulation syndrome: current views on pathophysiology, risk factors, prevention, and management. J Exp Clin Assist Reprod 2009;6:3.

4. Kol S, Solt I. GnRH agonist for triggering final oocyte maturation in patients at risk of ovarian hyperstimulation syndrome: still a controversy? J Assist Reprod Genet 2008;25:63-6.

5. Griesinger G, Diedrich K, Devroey P, Kolibianakis EM. $\mathrm{GnRH}$ agonist for triggering final oocyte maturation in 
the $\mathrm{GnRH}$ antagonist ovarian hyperstimulation protocol: a systematic review and meta-analysis. Hum Reprod Update 2006;12:159-68.

6. Kol S, Humaidan P. GnRH agonist triggering: recent developments. Reprod Biomed Online 2013;26:22630.

7. Kol S, Dor J. Prevention of OHSS: GnRH agonist versus HCG to trigger ovulation. Reprod Biomed Online 2009;19:59-60.
8. Engmann L, Siano L, Schmidt D, Nulsen J, Maier D, Benadiva C. GnRH agonist to induce oocyte maturation during IVF in patients at high risk of OHSS. Reprod Biomed Online 2006;13:639-44.

9. Shapiro BS, Daneshmand ST, Garner FC, Aguirre M, Hudson C. Comparison of "triggers" using leuprolide acetate alone or in combination with low-dose human chorionic gonadotropin. Fertil Steril 2011;95:2715-7. 